\title{
Existence of solutions for Sturm-Liouville boundary value problems of higher-order coupled fractional differential equations at resonance
}

\author{
Tingting Xue ${ }^{1,2}$, Wenbin Liu ${ }^{1 *}$ and Wei Zhang ${ }^{1}$
}

"Correspondence:

wblium@163.com

'Department of Mathematics, China

University of Mining and

Technology, Xuzhou, 221116, P.R.

China

Full list of author information is

available at the end of the article

\begin{abstract}
This paper focuses on the existence of solutions for a higher-order coupled system of fractional differential equations with Sturm-Liouville boundary value conditions at resonance. By applying Mawhin continuation theorem, some new existence results are established. Furthermore, two examples are supplied to demonstrate the main results.
\end{abstract}

MSC: $34 \mathrm{~A} 08 ; 34 \mathrm{~B} 15$

Keywords: fractional differential system; Sturm-Liouville boundary value conditions; resonance; Mawhin continuation theorem

\section{Introduction}

In the last two decades, fractional differential equations have been widely used in various fields of science, engineering and mathematics (see [1-7]). Based on the extensive application of fractional differential equations, it is of great theoretical and practical significance to study the boundary value problems (BVPs for short) of fractional differential equations. Accordingly, the research of fractional BVPs has been paid attention to by many scholars. At present, there have been many studies on various BVPs of fractional differential equations, for example, periodic BVPs (see [8-10]), anti-periodic BVPs (see [11, 12]), Dirichlet BVPs (see [13, 14]), multi-point BVPs (see [15-17]), impulsive BVPs (see [18]), Sturm-Liouville BVPs (see [19-22]), etc. Among them, as a classical non-resonance boundary value condition, the integer order Sturm-Liouville BVPs have been studied for a long time. By comparison, the study of fractional Sturm-Liouville BVPs is still a new field. In [23], Zhao considered the following fractional Sturm-Liouville BVP:

$$
\left\{\begin{array}{l}
D_{0+}^{\alpha} u(t)+f(t, u(t))=0, \quad 0<t<1, \\
u^{\prime}(0)-\beta u(\xi)=0, \quad u^{\prime}(1)+\gamma u(\eta)=0,
\end{array}\right.
$$

where $1<\alpha \leq 2, D_{0+}^{\alpha}$ is the Caputo fractional derivative, $0 \leq \xi \leq \eta \leq 1,0 \leq \beta, \gamma \leq 1$, $f:[0,1] \times \mathbb{R}^{+} \rightarrow \mathbb{R}^{+}$is continuous. On the basis of fixed point theorems and successive

(c) The Author(s) 2017. This article is distributed under the terms of the Creative Commons Attribution 4.0 International License (http://creativecommons.org/licenses/by/4.0/), which permits unrestricted use, distribution, and reproduction in any medium, provided you give appropriate credit to the original author(s) and the source, provide a link to the Creative Commons license, and indicate if changes were made. 
iteration method, the existence of positive solutions was obtained. On this foundation, Liu [24] studied such problems further by applying the Avery-Peterson fixed point theorem.

Zhao [25] studied the existence of at least two positive solutions by the fixed point theorem in the cone of strict-set-contraction operators for the following BVP:

$$
\left\{\begin{array}{l}
D_{0+}^{q} u(t)+f\left(t, u, u^{\prime}, \ldots, u^{(n-2)}\right)=0, \quad 0<t<1 \\
u(0)=u^{\prime}(0)=\cdots=u^{(n-3)}(0)=0, \\
\alpha u^{(n-2)}(0)-\beta u^{(n-1)}(0)=0, \quad \gamma u^{(n-2)}(1)+\delta u^{(n-1)}(1)=0
\end{array}\right.
$$

where $n-1<q \leq n, D_{0+}^{q}$ is the Caputo fractional derivative, $n \geq 2, \alpha, \beta, \gamma$ and $\delta$ are nonnegative constants. Due to the widespread use of coupled system in the applications (see [26-28]), it is important to study a coupled system of fractional differential equations and some important results have been presented (see [29-32]). Zhang and Bai [33] studied the following equations:

$$
\left\{\begin{array}{l}
D_{0+}^{\alpha} u(t)=f\left(t, v(t), D_{0+}^{\beta-1} v(t)\right), \quad 0<t<1, \\
D_{0+}^{\beta} v(t)=g\left(t, u(t), D_{0+}^{\alpha-1} u(t)\right), \quad 0<t<1, \\
u(0)=v(0)=0, \quad u(1)=\sigma_{1} u\left(\eta_{1}\right), \quad v(1)=\sigma_{2} v\left(\eta_{2}\right),
\end{array}\right.
$$

where $D_{0_{+}}^{\alpha}, D_{0_{+}}^{\beta}$ are Riemann-Liouville fractional derivatives, $1<\alpha, \beta \leq 2,0<\eta_{1}, \eta_{2}<1$, $\sigma_{1}, \sigma_{2}>0, f, g:[0,1] \times \mathbb{R}^{2} \rightarrow \mathbb{R}$ are continuous. The sufficient conditions for the existence of solutions of coupled fractional differential equations are obtained by applying the Mawhin continuation theorem.

Recently, there have appeared some papers dealing with the existence of solutions for a coupled system of higher-order fractional differential equations (see [34-38]). However, there are few results concerning a higher-order coupled system of fractional differential equations with Sturm-Liouville boundary value conditions at resonance. Motivated by the above mentioned discussion, we consider the following BVP:

$$
\left\{\begin{array}{l}
D_{0+}^{\alpha} u(t)=f\left(t, v(t), v^{\prime}(t), \ldots, v^{(n-1)}(t)\right), \\
D_{0+}^{\beta} v(t)=g\left(t, u(t), u^{\prime}(t), \ldots, u^{(n-1)}(t)\right), \\
u(0)=u^{\prime}(0)=\cdots=u^{(n-3)}(0)=0, \\
v(0)=v^{\prime}(0)=\cdots=v^{(n-3)}(0)=0, \\
u^{(n-2)}(0)=\gamma_{1} u^{(n-1)}\left(\xi_{1}\right), \quad u^{(n-1)}(1)=\delta_{1} u^{(n-2)}\left(\eta_{1}\right), \\
v^{(n-2)}(0)=\gamma_{2} v^{(n-1)}\left(\xi_{2}\right), \quad v^{(n-1)}(1)=\delta_{2} v^{(n-2)}\left(\eta_{2}\right),
\end{array}\right.
$$

where $0<t<1, n-1<\alpha, \beta \leq n, n>2, D_{0_{+}}^{\alpha}, D_{0_{+}}^{\beta}$ are Caputo fractional derivatives, $f, g$ : $[0,1] \times \mathbb{R}^{n} \rightarrow \mathbb{R}$ are continuous, $\gamma_{1}, \gamma_{2}, \delta_{1}, \delta_{2}>0,0<\xi_{1}, \xi_{2}, \eta_{1}, \eta_{2}<1$. Equation (1.1) is a Sturm-Liouville semi-homogeneous BVP.

The major contributions given in this paper have some new features. Firstly, the fractional differential equation established by us is a higher-order coupled system which is more difficult to construct than a projection operator. In comparison with the previous literature, the system is more general. Secondly, we also observe that few scholars have ever considered the higher-order coupled system of fractional differential equations with 
Sturm-Liouville boundary value conditions at resonance before. So our results serve as a further development for previous findings in this sense. In addition, we can also use the method of this paper to discuss the following BVP, which is similar to (1.1):

$$
\left\{\begin{array}{l}
D_{0+}^{\alpha} u(t)=f\left(t, v(t), v^{\prime}(t), \ldots, v^{(n-1)}(t)\right), \\
D_{0+}^{\beta} v(t)=g\left(t, u(t), u^{\prime}(t), \ldots, u^{(n-1)}(t)\right), \\
u(0)=u^{\prime}(0)=\cdots=u^{(n-3)}(0)=0, \\
v(0)=v^{\prime}(0)=\cdots=v^{(n-3)}(0)=0, \\
u^{(n-2)}(1)=\gamma_{1} u^{(n-1)}\left(\xi_{1}\right), \quad u^{(n-1)}(0)=\delta_{1} u^{(n-2)}\left(\eta_{1}\right), \\
v^{(n-2)}(1)=\gamma_{2} v^{(n-1)}\left(\xi_{2}\right), \quad v^{(n-1)}(0)=\delta_{2} v^{(n-2)}\left(\eta_{2}\right) .
\end{array}\right.
$$

\section{Preliminaries}

In order to facilitate understanding, we introduce the concepts and lemmas of fractional derivatives and integrals related to this paper, and more details can be found in the recent literature (see [39-41]).

Definition 2.1 ([39]) Let $X, Y$ be real Banach spaces, and $L: \operatorname{dom} L \subset X \rightarrow Y$ be a linear map. If $\operatorname{dim} \operatorname{Ker} L=\operatorname{codim} \operatorname{Im} L<+\infty$ and $\operatorname{Im} L$ is a closed subset in $Y$, then the map $L$ is a Fredholm operator with index zero. If there exists the continuous projections $P: X \rightarrow X$ and $Q: Y \rightarrow Y$ satisfying $\operatorname{Im} P=\operatorname{Ker} L$ and $\operatorname{Ker} Q=\operatorname{Im} L$, then $\left.L\right|_{\operatorname{dom} L \cap \operatorname{Ker} P}:$ $\operatorname{dom} L \cap \operatorname{Ker} P \rightarrow \operatorname{Im} L$ is reversible. We denote the inverse of this map by $K_{P}$, i.e. $K_{P}=L_{P}^{-1}$ and $K_{P, Q}=K_{P}(I-Q)$. If $\Omega$ is an open bounded subset of $X$ and $\operatorname{dom} L \cap \Omega \neq \varnothing$, then the map $N$ is $L$-compact on $\bar{\Omega}$ when $Q N: \bar{\Omega} \rightarrow Y$ is bounded and $K_{P}(I-Q) N: \bar{\Omega} \rightarrow X$ is compact.

Theorem 2.1 ([39]) Let L be a Fredholm operator of index zero and $N$ be L-compact on $\bar{\Omega}$. Assume that the following conditions are satisfied:

(a 1$) L x \neq \lambda N x$ for any $(x, \lambda) \in[(\operatorname{dom} L \backslash \operatorname{Ker} L) \cap \partial \Omega] \times(0,1)$;

$\left(a_{2}\right) N x \notin \operatorname{Im} L$ for any $x \in \operatorname{Ker} L \cap \partial \Omega$;

$\left(a_{3}\right) \operatorname{deg}\left(\left.Q N\right|_{\operatorname{Ker} L}, \Omega \cap \operatorname{Ker} L, 0\right) \neq 0$.

Then the equation $L x=N x$ has at least one solution in $\operatorname{dom} L \cap \Omega$.

Definition 2.2 ([40]) The Riemann-Liouville fractional integral of order $\alpha(\alpha>0)$ for the function $x:(0,+\infty) \rightarrow \mathbb{R}$ is defined as

$$
I_{0+}^{\alpha} x(t)=\frac{1}{\Gamma(\alpha)} \int_{0}^{t}(t-s)^{\alpha-1} x(s) d s
$$

provided that the right-hand side integral is defined on $(0,+\infty)$.

Definition 2.3 ([41]) The Caputo fractional integral of order $\alpha(\alpha>0)$ for the function $x:(0,+\infty) \rightarrow \mathbb{R}$ is defined as

$$
{ }^{C} D_{0+}^{\alpha} x(t)=I_{0+}^{n-\alpha} \frac{d^{n} x(t)}{d t^{n}}=\frac{1}{\Gamma(n-\alpha)} \int_{0}^{t}(t-s)^{n-\alpha-1} x^{(n)}(s) d s,
$$

where $n=[\alpha]+1$, provided that the right-hand side integral is defined on $(0,+\infty)$. 
Lemma 2.1 ([41]) Let $n-1<\alpha \leq n$, if ${ }^{C} D_{0+}^{\alpha} x(t) \in C[0,1]$, then

$$
I_{0+}^{\alpha}{ }^{C} D_{0+}^{\alpha} x(t)=x(t)+c_{0}+c_{1} t+c_{2} t^{2}+\cdots+c_{n-1} t^{n-1},
$$

where $c_{i} \in \mathbb{R}, i=0,1, \ldots, n-1, n=[\alpha]+1$.

Lemma 2.2 ([41]) If $n-1<\alpha \leq n$, then the fractional differential ${ }^{C} D_{0+}^{\alpha} x(t)=0$ has the following form:

$$
x(t)=c_{0}+c_{1} t+c_{2} t^{2}+\cdots+c_{n-1} t^{n-1},
$$

where $c_{i} \in \mathbb{R}, i=0,1, \ldots, n-1, n=[\alpha]+1$.

Lemma 2.3 ([41]) Let $x(t) \in C[0,1], \alpha>0$, then

$$
{ }^{C} D_{0+}^{\alpha} I_{0+}^{\alpha} x(t)=x(t) .
$$

Lemma 2.4 ([41]) Let $\alpha>\beta>0$, then for any $x \in C[0,1] \cap L^{1}[0,1]$,

$$
{ }^{C} D_{0+}^{\beta} I_{0+}^{\alpha} x(t)=I_{0+}^{\alpha-\beta} x(t) .
$$

Lemma 2.5 ([41]) Let $x(t) \in L[0,1], \alpha, \beta>0$, then

$$
I_{0+}^{\alpha} I_{0+}^{\beta} x(t)=I_{0+}^{\alpha+\beta} x(t) .
$$

\section{Main result}

In order to prove the solvability of BVP (1.1), some notations are introduced.

In this paper, we define $X=C^{n-1}[0,1]$ with the norm $\|x\|_{X}=\max \left\{\|x\|_{\infty},\left\|x^{\prime}\right\|_{\infty}, \ldots\right.$, $\left.\left\|x^{(n-1)}\right\|_{\infty}\right\}$ and $Y=C[0,1]$ with the norm $\|y\|_{Y}=\|y\|_{\infty}$, where $\|x\|_{\infty}=\max _{t \in[0,1]}|x(t)|$. It is clear that $\left(X,\|\cdot\|_{X}\right)$ and $\left(Y,\|\cdot\|_{Y}\right)$ are Banach space. Furthermore, we consider Banach space $\bar{X}=X \times X$ with the norm $\|(u, v)\|_{\bar{X}}=\max \left\{\|u\|_{X},\|v\|_{X}\right\}$ and $\bar{Y}=Y \times Y$ with the norm $\|(x, y)\|_{\bar{Y}}=\max \left\{\|x\|_{Y},\|y\|_{Y}\right\}$.

Define the linear operator $L_{1}: \operatorname{dom} L_{1} \subset X \rightarrow Y$ as

$$
L_{1} u=D_{0+}^{\alpha} u,
$$

where

$$
\begin{aligned}
\operatorname{dom} L_{1}= & \left\{u \in X \mid D_{0+}^{\alpha} u(t) \in Y, u(0)=u^{\prime}(0)=\cdots=u^{(n-3)}(0)=0,\right. \\
& \left.u^{(n-2)}(0)=\gamma_{1} u^{(n-1)}\left(\xi_{1}\right), u^{(n-1)}(1)=\delta_{1} u^{(n-2)}\left(\eta_{1}\right)\right\} .
\end{aligned}
$$

Define the linear operator $L_{2}: \operatorname{dom} L_{2} \subset X \rightarrow Y$ as

$$
L_{2} v=D_{0+}^{\beta} v,
$$

where

$$
\begin{aligned}
\operatorname{dom} L_{2}= & \left\{v \in X \mid D_{0+}^{\beta} v(t) \in Y, v(0)=v^{\prime}(0)=\cdots=v^{(n-3)}(0)=0,\right. \\
& \left.v^{(n-2)}(0)=\gamma_{2} v^{(n-1)}\left(\xi_{2}\right), v^{(n-1)}(1)=\delta_{2} v^{(n-2)}\left(\eta_{2}\right)\right\} .
\end{aligned}
$$


Define the operator $L: \operatorname{dom} L \subset \bar{X} \rightarrow \bar{Y}$ as

$$
L(u, v)=\left(L_{1} u, L_{2} v\right)
$$

where $\operatorname{dom} L=\left\{(u, v) \in \bar{X} \mid u \in \operatorname{dom} L_{1}, v \in \operatorname{dom} L_{2}\right\}$, and we define $N: \bar{X} \rightarrow \bar{Y}$ by setting

$$
N(u, v)=\left(N_{1} v, N_{2} u\right)
$$

where $N_{1}: Y \rightarrow X$ is defined as

$$
N_{1} v(t)=f\left(t, v(t), v^{\prime}(t), \ldots, v^{(n-1)}(t)\right)
$$

and $N_{2}: Y \rightarrow X$ is defined as

$$
N_{2} u(t)=g\left(t, u(t), u^{\prime}(t), \ldots, u^{(n-1)}(t)\right)
$$

Then BVP (1.1) is equivalent to the following operator equation:

$$
L(u, v)=N(u, v), \quad(u, v) \in \operatorname{dom} L .
$$

Next we establish the existence results for BVP (1.1) in the following cases:

Case (i) $\gamma_{1}=\gamma_{2}=0, \delta_{1} \eta_{1}=\delta_{2} \eta_{2}=1$;

Case (ii) $\left(\gamma_{1}+\eta_{1}\right) \delta_{1}=\left(\gamma_{2}+\eta_{2}\right) \delta_{2}=1$.

Firstly, the main conclusions of Case (i) are given as follows.

Theorem 3.1 For Case (i), assume that the following conditions hold.

$\left(\mathrm{H}_{1}\right)$ If the functions $f, g:[0,1] \times \mathbb{R}^{n} \rightarrow \mathbb{R}$ satisfy the Carathéodary condition, and there exist nonnegative functions $a_{i}, d_{i}, b_{1}, b_{2}, r_{1}, r_{2} \in Y$ and constant $\theta_{1}, \theta_{2} \in[0,1), i=\overline{0, n-1}$, for $\forall\left(x_{0}, x_{1}, \ldots, x_{n-1}\right) \in \mathbb{R}^{n}, t \in[0,1]$, the following inequalities hold:

$$
\begin{aligned}
\left|f\left(t, x_{0}, x_{1}, x_{2}, \ldots, x_{n-1}\right)\right| & \leq \sum_{i=0}^{n-1} a_{i}(t)\left|x_{i}\right|+b_{1}(t) \sum_{i=0}^{n-1}\left|x_{i}\right|^{\theta_{1}}+r_{1}(t), \\
\left|g\left(t, x_{0}, x_{1}, x_{2}, \ldots, x_{n-1}\right)\right| & \leq \sum_{i=0}^{n-1} d_{i}(t)\left|x_{i}\right|+b_{2}(t) \sum_{i=0}^{n-1}\left|x_{i}\right|^{\theta_{2}}+r_{2}(t) .
\end{aligned}
$$

$\left(\mathrm{H}_{2}\right)$ There exists a constant $M>0$ such that for $\forall t \in[0,1]$, if $\left|u^{(n-1)}(t)\right|>M$ and $\left|v^{(n-1)}(t)\right|>$ $M$, then $Q N(u, v) \neq(0,0)$.

$\left(\mathrm{H}_{3}\right)$ There exists a constant $M^{*}>0$ such that for $\forall c_{1}, c_{2} \in \mathbb{R}$ satisfying $\min \left\{\left|c_{1}\right|,\left|c_{2}\right|\right\}>M^{*}$, one has either

$$
c_{1} N_{1}\left(c_{2} t^{n-1}\right)>0, \quad c_{2} N_{2}\left(c_{1} t^{n-1}\right)>0
$$

or

$$
c_{1} N_{1}\left(c_{2} t^{n-1}\right)<0, \quad c_{2} N_{2}\left(c_{1} t^{n-1}\right)<0
$$


$\left(\mathrm{H}_{4}\right) \max \left\{2 a_{1} \sum_{i=0}^{n-1}\left\|a_{i}\right\|_{\infty}, a_{1} \sum_{i=0}^{n-1}\left\|a_{i}\right\|_{\infty}+a_{2} \sum_{i=0}^{n-1}\left\|d_{i}\right\|_{\infty}, 2 a_{2} \sum_{i=0}^{n-1}\left\|d_{i}\right\|_{\infty}\right\}<1$, where $a_{1}=\frac{1}{\Gamma(\alpha-n+2)}, a_{2}=\frac{1}{\Gamma(\beta-n+2)}$.

Then BVP (1.1) has at least one solution.

To prove the above theorem, we begin with the following lemmas.

Lemma 3.1 Let L be defined by (3.1), then

$$
\begin{aligned}
& \operatorname{Ker} L=\left(\operatorname{Ker} L_{1}, \operatorname{Ker} L_{2}\right)=\left\{(u, v) \in \bar{X} \mid(u, v)=\left(c_{1} t^{n-1}, c_{2} t^{n-1}\right), c_{1}, c_{2} \in \mathbb{R}\right\} \\
& \operatorname{Im} L=\left(\operatorname{Im} L_{1}, \operatorname{Im} L_{2}\right)=\left\{(x, y) \in \bar{Y} \mid T_{1} x=0, T_{2} y=0\right\}
\end{aligned}
$$

where

$$
\begin{aligned}
& T_{1} x=\int_{0}^{1}(1-s)^{\alpha-n} x(s) d s-\frac{\delta_{1}}{\alpha-n+1} \int_{0}^{\eta_{1}}\left(\eta_{1}-s\right)^{\alpha-n+1} x(s) d s, \\
& T_{2} y=\int_{0}^{1}(1-s)^{\beta-n} y(s) d s-\frac{\delta_{2}}{\beta-n+1} \int_{0}^{\eta_{2}}\left(\eta_{2}-s\right)^{\beta-n+1} y(s) d s .
\end{aligned}
$$

Proof According to Lemma 2.2, $L_{1} u=D_{0+}^{\alpha} u(t)=0$ has the solution

$$
u(t)=c_{0}+c_{1} t+c_{2} t^{2}+\cdots+c_{n-1} t^{n-1}, \quad c_{i} \in \mathbb{R}, i=\overline{0, n-1}
$$

By the definition of $\operatorname{dom} L_{1}$, we have $c_{i}=0, i=\overline{0, n-2}$, thus

$$
\operatorname{Ker} L_{1}=\left\{u \in X \mid u(t)=c_{1} t^{n-1}, \forall t \in[0,1], c_{1} \in \mathbb{R}\right\}
$$

For $x \in \operatorname{Im} L_{1}$, there exists $u \in \operatorname{dom} L_{1}$ such that $x=L_{1} u \in Y$. From Lemma 2.1, we have

$$
u(t)=I_{0+}^{\alpha} x(t)+c_{0}+c_{1} t+c_{2} t^{2}+\cdots+c_{n-1} t^{n-1}
$$

Then by the definition of $\operatorname{dom} L_{1}$ we have $c_{i}=0, i=\overline{0, n-2}$. Hence

$$
u(t)=I_{0+}^{\alpha} x(t)+c_{n-1} t^{n-1}
$$

According to Lemma 2.4, we obtain

$$
\begin{aligned}
& u^{(n-1)}(t)=I_{0+}^{\alpha-n+1} x(t)+c_{n-1}(n-1) ! \\
& u^{(n-2)}(t)=I_{0+}^{\alpha-n+2} x(t)+c_{n-1}(n-1) ! t
\end{aligned}
$$

Taking into account the boundary condition $u^{(n-1)}(1)=\delta_{1} u^{(n-2)}\left(\eta_{1}\right)$ and $\delta_{1} \eta_{1}=1$ of Case (i), we see that $x$ satisfies

$$
T_{1} x=\int_{0}^{1}(1-s)^{\alpha-n} x(s) d s-\frac{\delta_{1}}{\alpha-n+1} \int_{0}^{\eta_{1}}\left(\eta_{1}-s\right)^{\alpha-n+1} x(s) d s=0 .
$$

On the other hand, assume that $x \in Y$ satisfies the equation $T_{1} x=0$. Let $u(t)=I_{0+}^{\alpha} x(t)$, then $u \in \operatorname{dom} L_{1}$. By Lemma 2.3, we have $D_{0+}^{\alpha} u(t)=x(t)$, so $x \in \operatorname{Im} L_{1}$. Then we get

$$
\operatorname{Im} L_{1}=\left\{x \in Y \mid T_{1} x=0\right\}
$$


Similarly, we have

$$
\begin{aligned}
& \operatorname{Ker} L_{2}=\left\{v \in X \mid v(t)=c_{2} t^{n-1}, \forall t \in[0,1], c_{2} \in \mathbb{R}\right\} \\
& \operatorname{Im} L_{2}=\left\{y \in Y \mid T_{2} y=0\right\}
\end{aligned}
$$

Then, the proof is complete.

Lemma 3.2 Let $L$ be defined by (3.1), then $L$ is a Fredholm operator of index zero. The linear continuous projector operators $P: \bar{X} \rightarrow \bar{X}$ and $Q: \bar{Y} \rightarrow \bar{Y}$ can be defined as

$$
\begin{aligned}
& P(u, v)=\left(P_{1} u, P_{2} v\right)=\left(\frac{u^{(n-1)}(0)}{(n-1) !} t^{n-1}, \frac{v^{(n-1)}(0)}{(n-1) !} t^{n-1}\right) \\
& Q(x, y)=\left(Q_{1} x, Q_{2} y\right)=\left(\frac{\alpha-n+1}{1-\frac{\eta_{1} \alpha-n+1}{\alpha-n+2}} T_{1} x, \frac{\beta-n+1}{1-\frac{\eta_{2} \beta-n+1}{\beta-n+2}} T_{2} y\right),
\end{aligned}
$$

and $K_{P}: \operatorname{Im} L \rightarrow \operatorname{dom} L \cap \operatorname{Ker} P$ by

$$
K_{P}(x, y)=\left(I_{0+}^{\alpha} x(t), I_{0+}^{\beta} y(t)\right)
$$

Proof Obviously $\operatorname{Im} P=\operatorname{Ker} L$ and $P^{2}(u, v)=P(u, v)$. Since $(u, v)=((u, v)-P(u, v))+P(u, v)$, it is clear that $\bar{X}=\operatorname{Ker} P+\operatorname{Ker} L$. By calculation, we get $\operatorname{Ker} L \cap \operatorname{Ker} P=\{(0,0)\}$. Thus, we obtain

$$
\bar{X}=\operatorname{Ker} L \oplus \operatorname{Ker} P \text {. }
$$

For every $(u, v) \in \bar{X}$, we have

$$
\|P(u, v)\|_{\bar{X}} \leq \max \left\{\left|u^{(n-1)}(0)\right|,\left|v^{(n-1)}(0)\right|\right\} .
$$

Taking $(x, y) \in \bar{Y}$, one has

$$
Q^{2}(x, y)=Q\left(Q_{1} x, Q_{2} y\right)=\left(Q_{1}^{2} x, Q_{2}^{2} y\right)
$$

By the definition of $Q_{1}$, we obtain

$$
Q_{1}^{2} x=Q_{1} x \cdot \frac{\alpha-n+1}{1-\frac{\eta_{1} \alpha-n+1}{\alpha-n+2}}\left(\int_{0}^{1}(1-s)^{\alpha-n} d s-\frac{\delta_{1}}{\alpha-n+1} \int_{0}^{\eta_{1}}\left(\eta_{1}-s\right)^{\alpha-n+1} d s\right)=Q_{1} x
$$

where for the denominator $1-\frac{\eta_{1}^{\alpha-n+1}}{\alpha-n+2}>0$ can be verified.

Similarly, $Q_{2}^{2} y=Q_{2} y$. This gives $Q^{2}(x, y)=Q(x, y)$. Let $(x, y)=((x, y)-Q(x, y))+Q(x, y)$, where $(x, y)-Q(x, y) \in \operatorname{Ker} Q=\operatorname{Im} L, Q(x, y) \in \operatorname{Im} Q$. It follows from $\operatorname{Ker} Q=\operatorname{Im} L$ and $Q^{2}(x, y)=Q(x, y)$ that $\operatorname{Im} Q \cap \operatorname{Im} L=\{(0,0)\}$. Then, we have

$$
\bar{Y}=\operatorname{Im} L \oplus \operatorname{Im} Q
$$

Thus

$$
\operatorname{dim} \operatorname{Ker} L=\operatorname{dim} \operatorname{Im} Q=\operatorname{codim} \operatorname{Im} L .
$$


This means that $L$ is a Fredholm operator of index zero.

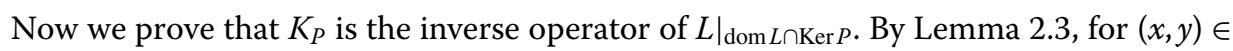
$\operatorname{Im} L$, we obtain

$$
L K_{P}(x, y)=\left(D_{0+}^{\alpha}\left(I_{0+}^{\alpha} x\right), D_{0+}^{\beta}\left(I_{0+}^{\beta} y\right)\right)=(x, y) .
$$

Moreover, for $(u, v) \in \operatorname{dom} L \cap \operatorname{Ker} P$, we have $u^{(n-1)}(0)=v^{(n-1)}(0)=0$. Together with the boundary condition, we get

$$
K_{P} L(u, v)=\left(I_{0+}^{\alpha} D_{0+}^{\alpha} u(t), I_{0+}^{\beta} D_{0+}^{\beta} v(t)\right)=(u, v) .
$$

To summarize, $K_{P}=\left(\left.L\right|_{\operatorname{dom} L \cap \operatorname{Ker} P}\right)^{-1}$.

Hence, for each $(x, y) \in \operatorname{Im} L$, by the definition of $\|\cdot\|_{\bar{X}}$ we have

$$
\begin{aligned}
\left\|K_{P}(x, y)\right\|_{\bar{X}} & \leq \max \left\{\frac{1}{\Gamma(\alpha-n+2)}\|x\|_{\infty}, \frac{1}{\Gamma(\beta-n+2)}\|y\|_{\infty}\right\} \\
& :=\max \left\{a_{1}\|x\|_{\infty}, a_{2}\|y\|_{\infty}\right\},
\end{aligned}
$$

where $a_{1}=\frac{1}{\Gamma(\alpha-n+2)}, a_{2}=\frac{1}{\Gamma(\beta-n+2)}$. The proof is complete.

The main proof of Theorem 3.1 is given by the following three steps.

\section{Proof of Theorem 3.1}

Step 1 Let

$$
\Omega_{1}=\{(u, v) \in \operatorname{dom} L \backslash \operatorname{Ker} L \mid L(u, v)=\lambda N(u, v), \lambda \in(0,1)\} .
$$

For $(u, v) \in \Omega_{1}$, we have $L(u, v)=\lambda N(u, v) \in \operatorname{Im} L=\operatorname{Ker} Q$, thus $Q N(u, v)=(0,0)$, i.e. $Q_{1} N_{1} v(t)=0, Q_{2} N_{2} u(t)=0$. From $\left(\mathrm{H}_{2}\right)$, we know there exists $t_{0}, t_{1} \in(0,1)$, such that $\left|v^{(n-1)}\left(t_{0}\right)\right| \leq M$ and $\left|u^{(n-1)}\left(t_{1}\right)\right| \leq M$. It is easy to check that $\|u\|_{X}=\left\|u^{(n-1)}\right\|_{\infty},\|v\|_{X}=$ $\left\|v^{(n-1)}\right\|_{\infty}$ for all $u \in \operatorname{dom} L_{1}, v \in \operatorname{dom} L_{2}$. Again for $(u, v) \in \Omega_{1}$, then $(I-P)(u, v) \in \operatorname{dom} L \cap$ $\operatorname{Ker} P$ and $L P(u, v)=(0,0)$. Hence, from (3.3), we get

$$
\begin{aligned}
\|(I-P)(u, v)\|_{\bar{X}} & =\left\|K_{p} L(I-P)(u, v)\right\|_{\bar{X}}=\left\|K_{p} L(u, v)\right\|_{\bar{X}}=\left\|K_{p}\left(L_{1} u, L_{2} v\right)\right\|_{\bar{X}} \\
& \leq \max \left\{a_{1}\left\|N_{1} v\right\|_{\infty}, a_{2}\left\|N_{2} u\right\|_{\infty}\right\} .
\end{aligned}
$$

By $L_{1} u=\lambda N_{1} u$ and $u \in \operatorname{dom} L_{1}$, we have

$$
\begin{aligned}
u(t)= & \frac{\lambda}{\Gamma(\alpha)} \int_{0}^{t}(t-s)^{\alpha-1} f\left(s, v(s), v^{\prime}(s), \ldots, v^{(n-1)}(s)\right) d s \\
& -u(0)-u^{\prime}(0) t-\cdots-\frac{u^{(n-1)}(0)}{(n-1) !} t^{n-1} .
\end{aligned}
$$

Furthermore, we obtain

$$
u^{(n-1)}(t)=\frac{\lambda}{\Gamma(\alpha-n+1)} \int_{0}^{t}(t-s)^{\alpha-n} f\left(s, v(s), v^{\prime}(s), \ldots, v^{(n-1)}(s)\right) d s-u^{(n-1)}(0)
$$


then substituting $t=t_{1}$ into the above equation, we get

$$
u^{(n-1)}\left(t_{1}\right)=\frac{\lambda}{\Gamma(\alpha-n+1)} \int_{0}^{t_{1}}\left(t_{1}-s\right)^{\alpha-n} f\left(s, v(s), v^{\prime}(s), \ldots, v^{(n-1)}(s)\right) d s-u^{(n-1)}(0)
$$

Together with $\left|u^{(n-1)}\left(t_{1}\right)\right| \leq M$ and $\left(\mathrm{H}_{1}\right)$, we get

$$
\begin{aligned}
\left|u^{(n-1)}(0)\right| \leq & \left|\frac{\lambda}{\Gamma(\alpha-n+1)} \int_{0}^{t_{1}}\left(t_{1}-s\right)^{\alpha-n} f\left(s, v(s), v^{\prime}(s), \ldots, v^{(n-1)}(s)\right) d s\right|+\left|u^{(n-1)}\left(t_{1}\right)\right| \\
\leq & \frac{1}{\Gamma(\alpha-n+1)} \int_{0}^{t_{1}}\left(t_{1}-s\right)^{\alpha-n}\left|f\left(s, v(s), v^{\prime}(s), \ldots, v^{(n-1)}(s)\right)\right| d s+M \\
\leq & \frac{1}{\Gamma(\alpha-n+1)} \int_{0}^{t_{1}}\left(t_{1}-s\right)^{\alpha-n}\left(\sum_{i=0}^{n-1} a_{i}(t)\left|v^{(i)}\right|+b_{1}(t) \sum_{i=0}^{n-1}\left|v^{(i)}\right|^{\theta_{1}}+r_{1}(t)\right) d s \\
& +M \\
\leq & \frac{1}{\Gamma(\alpha-n+1)}\left(\sum_{i=0}^{n-1}\left\|a_{i}\right\|_{\infty}\left\|v^{(i)}\right\|_{\infty}+\left\|b_{1}\right\|_{\infty} \sum_{i=0}^{n-1}\left\|v^{(i)}\right\|_{\infty}^{\theta_{1}}+\left\|r_{1}\right\|_{\infty}\right) \\
& \times \int_{0}^{t_{1}}\left(t_{1}-s\right)^{\alpha-n} d s+M \\
\leq & a_{1}\left(\sum_{i=0}^{n-1}\left\|a_{i}\right\|_{\infty}\left\|v^{(i)}\right\|_{\infty}+\left\|b_{1}\right\|_{\infty} \sum_{i=0}^{n-1}\left\|v^{(i)}\right\|_{\infty}^{\theta_{1}}+\left\|r_{1}\right\|_{\infty}\right)+M,
\end{aligned}
$$

where $a_{1}=\frac{1}{\Gamma(\alpha-n+2)}$. Similarly, we obtain

$$
\left|v^{(n-1)}(0)\right| \leq a_{2}\left(\sum_{i=0}^{n-1}\left\|d_{i}\right\|_{\infty}\left\|u^{(i)}\right\|_{\infty}+\left\|b_{2}\right\|_{\infty} \sum_{i=0}^{n-1}\left\|u^{(i)}\right\|_{\infty}^{\theta_{2}}+\left\|r_{2}\right\|_{\infty}\right)+M,
$$

where $a_{2}=\frac{1}{\Gamma(\beta-n+2)}$. Combined with (3.2) and (3.4), we get

$$
\begin{aligned}
\|(u, v)\|_{\bar{X}}= & \|P(u, v)+(I-P)(u, v)\|_{\bar{X}} \\
\leq & \|P(u, v)\|_{\bar{X}}+\|(I-P)(u, v)\|_{\bar{X}} \\
\leq & \max \left\{\left|u^{(n-1)}(0)\right|+a_{1}\left\|N_{1} v\right\|_{\infty},\left|u^{(n-1)}(0)\right|+a_{2}\left\|N_{2} u\right\|_{\infty},\right. \\
& \left.\left|v^{(n-1)}(0)\right|+a_{1}\left\|N_{1} v\right\|_{\infty},\left|v^{(n-1)}(0)\right|+a_{2}\left\|N_{2} u\right\|_{\infty}\right\} .
\end{aligned}
$$

Next, we will prove this conclusion in four cases.

Case $1\|(u, v)\|_{\bar{X}} \leq\left|u^{(n-1)}(0)\right|+a_{1}\left\|N_{1} v\right\|_{\infty}$. 
By (3.5) and $\left(\mathrm{H}_{1}\right)$, we get

$$
\begin{aligned}
\|(u, v)\|_{\bar{X}} \leq & \left|u^{(n-1)}(0)\right|+a_{1}\left\|N_{1} v\right\|_{\infty} \\
\leq & a_{1}\left(\sum_{i=0}^{n-1}\left\|a_{i}\right\|_{\infty}\left\|v^{(i)}\right\|_{\infty}+\left\|b_{1}\right\|_{\infty} \sum_{i=0}^{n-1}\left\|v^{(i)}\right\|_{\infty}^{\theta_{1}}+\left\|r_{1}\right\|_{\infty}\right)+M \\
& +a_{1}\left\|f\left(t, v(t), v^{\prime}(t), \ldots, v^{(n-1)}(t)\right)\right\|_{\infty} \\
\leq & 2 a_{1}\left(\sum_{i=0}^{n-1}\left\|a_{i}\right\|_{\infty}\left\|v^{(i)}\right\|_{\infty}+\left\|b_{1}\right\|_{\infty} \sum_{i=0}^{n-1}\left\|v^{(i)}\right\|_{\infty}^{\theta_{1}}+\left\|r_{1}\right\|_{\infty}\right)+M \\
\leq & 2 a_{1}\left(\sum_{i=0}^{n-1}\left\|a_{i}\right\|_{\infty}\left\|v^{(i)}\right\|_{\infty}+n\left\|b_{1}\right\|_{\infty}\left\|v^{(n-1)}\right\|_{\infty}^{\theta_{1}}+\left\|r_{1}\right\|_{\infty}\right)+M \\
\leq & 2 a_{1}\left(\sum_{i=0}^{n-1}\left\|a_{i}\right\|_{\infty}\|v\|_{X}+n\left\|b_{1}\right\|_{\infty}\|v\|_{X}^{\theta_{1}}+\left\|r_{1}\right\|_{\infty}\right)+M .
\end{aligned}
$$

According to $\left(\mathrm{H}_{4}\right)$ and the definition of $\|(u, v)\|_{\bar{X}}$, from the above inequality, we can derive that $\|v\|_{X}$ is bounded. Therefore $\Omega_{1}$ is bounded.

Case $2\|(u, v)\|_{\bar{X}} \leq\left|v^{(n-1)}(0)\right|+a_{2}\left\|N_{2} u\right\|_{\infty}$. The proof is similar to Case 1 . Here, we omit it.

Case $3\|(u, v)\|_{\bar{X}} \leq\left|u^{(n-1)}(0)\right|+a_{2}\left\|N_{2} u\right\|_{\infty}$.

From (3.5) and $\left(\mathrm{H}_{1}\right)$, we get

$$
\begin{aligned}
\|(u, v)\|_{\bar{X}} \leq & \left|u^{(n-1)}(0)\right|+a_{2}\left\|N_{2} u\right\|_{\infty} \\
\leq & a_{1}\left(\sum_{i=0}^{n-1}\left\|a_{i}\right\|_{\infty}\left\|v^{(i)}\right\|_{\infty}+\left\|b_{1}\right\|_{\infty} \sum_{i=0}^{n-1}\left\|v^{(i)}\right\|_{\infty}^{\theta_{1}}+\left\|r_{1}\right\|_{\infty}\right)+M \\
& +a_{2}\left\|g\left(t, u(t), u^{\prime}(t), \ldots, u^{(n-1)}(t)\right)\right\|_{\infty} \\
\leq & a_{1}\left(\sum_{i=0}^{n-1}\left\|a_{i}\right\|_{\infty}\left\|v^{(i)}\right\|_{\infty}+\left\|b_{1}\right\|_{\infty} \sum_{i=0}^{n-1}\left\|v^{(i)}\right\|_{\infty}^{\theta_{1}}+\left\|r_{1}\right\|_{\infty}\right)+M \\
& +a_{2}\left(\sum_{i=0}^{n-1}\left\|d_{i}\right\|_{\infty}\left\|u^{(i)}\right\|_{\infty}+\left\|b_{2}\right\|_{\infty} \sum_{i=0}^{n-1}\left\|u^{(i)}\right\|_{\infty}^{\theta_{2}}+\left\|r_{2}\right\|_{\infty}\right) \\
\leq & a_{1}\left(\sum_{i=0}^{n-1}\left\|a_{i}\right\|_{\infty}\left\|v^{(n-1)}\right\|_{\infty}+n\left\|b_{1}\right\|_{\infty}\left\|v^{(n-1)}\right\|_{\infty}^{\theta_{1}}+\left\|r_{1}\right\|_{\infty}\right)+M \\
& +a_{2}\left(\sum_{i=0}^{n-1}\left\|d_{i}\right\|_{\infty}\left\|u^{(n-1)}\right\|_{\infty}+n\left\|b_{2}\right\|_{\infty}\left\|u^{(n-1)}\right\|_{\infty}^{\theta_{2}}+\left\|r_{2}\right\|_{\infty}\right) \\
& +a_{2}\left(\sum_{i=0}^{n-1}\left\|d_{i}\right\|_{\infty}\|u\|_{X}+n\left\|b_{2}\right\|_{\infty}\|u\|_{X}^{\theta_{2}}+\left\|r_{2}\right\|_{\infty}\right)+M . \\
= & a_{1}\left(\sum_{i=0}^{n-1}\left\|a_{i}\right\|_{\infty}\|v\|_{X}+n\left\|b_{1}\right\|_{\infty}\|v\|_{X}^{\theta_{1}}+\left\|r_{1}\right\|_{\infty}\right)
\end{aligned}
$$


By $\left(\mathrm{H}_{4}\right)$, from the above inequality, we see that $\|(u, v)\|_{\bar{X}}$ is bounded. Therefore $\Omega_{1}$ is bounded.

Case $4\|(u, v)\|_{\bar{X}} \leq\left|v^{(n-1)}(0)\right|+a_{1}\left\|N_{1} v\right\|_{\infty}$. The proof is similar to Case 3. Here, we omit it.

According to the above arguments, we prove that $\Omega_{1}$ is bounded.

Step 2 Let

$$
\Omega_{2}=\{(u, v) \mid(u, v) \in \operatorname{Ker} L, N(u, v) \in \operatorname{Im} L\} .
$$

For $(u, v) \in \Omega_{2}$, we have $(u, v)=\left(c_{1} t^{n-1}, c_{2} t^{n-1}\right), c_{1}, c_{2} \in \mathbb{R}$. In view of $N(u, v)=\left(N_{1} v, N_{2} u\right) \in$ $\operatorname{Im} L=\operatorname{Ker} Q$, we have $Q N(u, v)=(0,0)$, then $Q_{1} N_{1} v(t)=0, Q_{2} N_{2} u(t)=0$. Together with $\left(\mathrm{H}_{2}\right)$, there exist $t_{0}, t_{1} \in(0,1)$ such that $\left|v^{(n-1)}\left(t_{0}\right)\right| \leq M,\left|u^{(n-1)}\left(t_{1}\right)\right| \leq M$, which imply $\left|c_{i}\right| \leq$ $\frac{M}{(n-1) !}, i=1,2$. Thus, we get

$$
\|(u, v)\|_{\bar{X}} \leq M
$$

Hence, $\Omega_{2}$ is bounded.

Step 3 Let

$$
\Omega_{3}=\{(u, v) \in \operatorname{Ker} L \mid \lambda(u, v)+(1-\lambda) Q N(u, v)=(0,0), \lambda \in[0,1]\},
$$

for $(u, v) \in \Omega_{3}$, we get $(u, v)=\left(c_{1} t^{n-1}, c_{2} t^{n-1}\right), c_{1}, c_{2} \in \mathbb{R}$, and

$$
\begin{aligned}
& \lambda c_{1} t^{n-1}+(1-\lambda) Q_{1} N_{1}(v)=0, \\
& \lambda c_{2} t^{n-1}+(1-\lambda) Q_{2} N_{2}(u)=0,
\end{aligned}
$$

that is to say,

$$
\begin{aligned}
-\lambda c_{1}^{2} t^{n-1}= & (1-\lambda) c_{1} Q_{1} N_{1}(v) \\
= & (1-\lambda) c_{1} \frac{\alpha-n+1}{1-\frac{\eta_{1} \alpha-n+1}{\alpha-n+2}}\left(\int_{0}^{1}(1-s)^{\alpha-n} f\left(s, c_{2} s^{n-1}, c_{2}(n-1) s^{n-2}, \ldots, c_{2}(n-1) !\right) d s\right. \\
& \left.-\frac{\delta_{1}}{\alpha-n+1} \int_{0}^{\eta_{1}}\left(\eta_{1}-s\right)^{\alpha-n+1} f\left(s, c_{2} s^{n-1}, c_{2}(n-1) s^{n-2}, \ldots, c_{2}(n-1) !\right) d s\right), \\
-\lambda c_{2}^{2} t^{n-1}= & (1-\lambda) c_{2} Q_{2} N_{2}(u) \\
= & (1-\lambda) c_{2} \frac{\beta-n+1}{1-\frac{\eta_{2} \beta-n+1}{\beta-n+2}}\left(\int_{0}^{1}(1-s)^{\beta-n} g\left(s, c_{1} s^{n-1}, c_{1}(n-1) s^{n-2}, \ldots, c_{1}(n-1) !\right) d s\right. \\
& \left.-\frac{\delta_{2}}{\beta-n+1} \int_{0}^{\eta_{2}}\left(\eta_{2}-s\right)^{\alpha-n+1} g\left(s, c_{1} s^{n-1}, c_{1}(n-1) s^{n-2}, \ldots, c_{1}(n-1) !\right) d s\right) .
\end{aligned}
$$

If $\lambda=0$, then $Q_{1} N_{1}(v)=Q_{2} N_{2}(u)=0$, together with $\left(\mathrm{H}_{2}\right)$, we have $\left|u^{(n-1)}(t)\right| \leq M$, $\left|v^{(n-1)}(t)\right| \leq M$, which imply $\left|c_{i}\right| \leq \frac{M}{(n-1) !}, i=1,2$. If $\lambda \in(0,1]$, then $\left|c_{i}\right| \leq \frac{M}{(n-1) !}, i=1,2$. Otherwise, if $\left|c_{i}\right|>\frac{M}{(n-1) !}, i=1,2$, in view of the first part of $\left(\mathrm{H}_{3}\right)$, the left of the above two equations is less than 0 , while the right is greater than 0 , which is apparently contradictory. Thus, $\Omega_{3}$ is bounded. 
Let $\Omega$ is a bounded open set of $\bar{X}$, such that $\bigcup_{i=1}^{3} \overline{\Omega_{i}} \subset \Omega$. It follows from Lemma 3.2 that $L$ is a Fredholm operator of index zero. Based on the Arzela-Ascoli theorem, we obtain the result that $N$ is $L$-compact on $\bar{\Omega}$. By Step 1 and Step 2, we see that the following two conditions hold:

$\left(a_{1}\right) L(u, v) \neq \lambda N(u, v),((u, v), \lambda) \in[(\operatorname{dom} L \backslash \operatorname{Ker} L) \cap \partial \Omega] \times(0,1)$,

$\left(a_{2}\right) N x \notin \operatorname{Im} L,(u, v) \in \operatorname{Ker} L \cap \partial \Omega$.

Let

$$
H((u, v), \lambda)=\lambda(u, v)+(1-\lambda) Q N(u, v)
$$

According to Step 3, we get $H((u, v), \lambda) \neq 0$ for $(u, v) \in \operatorname{Ker} L \cap \partial \Omega$. Therefore,

$$
\begin{aligned}
\operatorname{deg}\left(\left.Q N\right|_{\operatorname{Ker} L}, \Omega \cap \operatorname{Ker} L,(0,0)\right) & =\operatorname{deg}(H(\cdot, 0), \Omega \cap \operatorname{Ker} L,(0,0)) \\
& =\operatorname{deg}(H(\cdot, 1), \Omega \cap \operatorname{Ker} L,(0,0)) \\
& =\operatorname{deg}(I, \Omega \cap \operatorname{Ker} L,(0,0)) \\
& \neq 0 .
\end{aligned}
$$

Hence, the condition $\left(a_{3}\right)$ of Theorem 2.1 is satisfied. By Theorem 2.1, we see that $L(u, v)=$ $N(u, v)$ has at least one set of fixed points in $\operatorname{dom} L \cap \bar{\Omega}$, so BVP (1.1) has at least one set of solutions. The proof is complete.

Remark 3.1 If the second part of $\left(\mathrm{H}_{3}\right)$ is satisfied, then the set

$$
\Omega_{3}^{\prime}=\{(u, v) \in \operatorname{Ker} L \mid-\lambda(u, v)+(1-\lambda) Q N(u, v)=(0,0), \lambda \in[0,1]\}
$$

is bounded.

Now we consider BVP (1.1) in the Case (ii); the main conclusion is given as follows.

Theorem 3.2 For Case (ii), assume that the following conditions hold.

$\left(\mathrm{H}_{1}\right)^{\prime}$ If the functions $f, g \in[0,1] \times \mathbb{R}^{n} \rightarrow \mathbb{R}$ satisfy the Carathéodary condition, and there exist nonnegative functions $a_{i}, d_{i}, b_{1}, b_{2}, r_{1}, r_{2} \in Y$ and constant $\theta_{1}, \theta_{2} \in[0,1), i=\overline{0, n-1}$, for $\forall\left(x_{0}, x_{1}, \ldots, x_{n-1}\right) \in \mathbb{R}^{n}, t \in[0,1]$, the following inequalities hold:

$$
\begin{aligned}
& \left|f\left(t, x_{0}, x_{1}, \ldots, x_{n-1}\right)\right| \leq \sum_{i=0}^{n-1} a_{i}(t)\left|x_{i}\right|+b_{1}(t) \sum_{i=0}^{n-1}\left|x_{i}\right|^{\theta_{1}}+r_{1}(t), \\
& \left|g\left(t, x_{0}, x_{1}, \ldots, x_{n-1}\right)\right| \leq \sum_{i=0}^{n-1} d_{i}(t)\left|x_{i}\right|+b_{2}(t) \sum_{i=0}^{n-1}\left|x_{i}\right|^{\theta_{2}}+r_{2}(t) .
\end{aligned}
$$

$\left(\mathrm{H}_{2}\right)^{\prime}$ There exists a constant $M>0$, such that, for $\forall t \in[0,1]$, if $\left|u^{(n-1)}(t)\right|>M$ and $\left|v^{(n-1)}(t)\right|>M$, then $Q N(u, v) \neq(0,0)$. 
$\left(\mathrm{H}_{3}\right)^{\prime}$ There exists a constant $M^{*}>0$ such that, for every $c_{1}, c_{2} \in \mathbb{R}$ satisfying $\min \left\{\left|c_{1}\right|,\left|c_{2}\right|\right\}>$ $M^{*}$, one has either

$$
c_{1} N_{1}\left(c_{2}\left(t^{n-1}+(n-1) \gamma_{2} t^{n-2}\right)\right)>0, \quad c_{2} N_{2}\left(c_{1}\left(t^{n-1}+(n-1) \gamma_{1} t^{n-2}\right)\right)>0,
$$

or

$$
c_{1} N_{1}\left(c_{2}\left(t^{n-1}+(n-1) \gamma_{2} t^{n-2}\right)\right)<0, \quad c_{2} N_{2}\left(c_{1}\left(t^{n-1}+(n-1) \gamma_{1} t^{n-2}\right)\right)<0 .
$$

$\left(\mathrm{H}_{4}\right)^{\prime}$

$$
\begin{gathered}
\max \left\{\left(2+n \gamma_{1}\right) a_{1} \sum_{i=0}^{n-1}\left\|a_{i}\right\|_{\infty},\left(1+(n-1) \gamma_{1}\right) a_{1} \sum_{i=0}^{n-1}\left\|a_{i}\right\|_{\infty}+\left(1+\gamma_{2}\right) a_{2} \sum_{i=0}^{n-1}\left\|d_{i}\right\|_{\infty},\right. \\
\left.\left(1+\gamma_{1}\right) a_{1} \sum_{i=0}^{n-1}\left\|a_{i}\right\|_{\infty}+\left(1+(n-1) \gamma_{2}\right) a_{2} \sum_{i=0}^{n-1}\left\|d_{i}\right\|_{\infty},\left(2+n \gamma_{2}\right) a_{2} \sum_{i=0}^{n-1}\left\|d_{i}\right\|_{\infty}\right\}<1,
\end{gathered}
$$

where $a_{1}=\frac{1}{\Gamma(\alpha-n+2)}, a_{2}=\frac{1}{\Gamma(\beta-n+2)}$.

Then BVP (1.1) has at least one solution.

To prove the above theorem, we have the following lemma, whose proof is similar to that of Lemma 3.1, Lemma 3.2 and is omitted.

Lemma 3.3 Let L be defined by (3.1), then

$$
\begin{aligned}
\operatorname{Ker} L= & \left(\operatorname{Ker} L_{1}, \operatorname{Ker} L_{2}\right) \\
= & \left\{(u, v) \in \bar{X} \mid(u, v)=\left(c_{1}\left(t^{n-1}+(n-1) \gamma_{1} t^{n-2}\right), c_{2}\left(t^{n-1}+(n-1) \gamma_{2} t^{n-2}\right)\right),\right. \\
& \left.c_{1}, c_{2} \in \mathbb{R}\right\}, \\
\operatorname{Im} L= & \left(\operatorname{Im} L_{1}, \operatorname{Im} L_{2}\right)=\left\{(x, y) \in \bar{Y} \mid T_{3} x=0, T_{4} y=0\right\},
\end{aligned}
$$

where

$$
\begin{aligned}
T_{3} x= & \int_{0}^{1}(1-s)^{\alpha-n} x(s) d s-\frac{\delta_{1}}{\alpha-n+1} \int_{0}^{\eta_{1}}\left(\eta_{1}-s\right)^{\alpha-n+1} x(s) d s \\
& -\gamma_{1} \delta_{1} \int_{0}^{\xi_{1}}\left(\xi_{1}-s\right)^{\alpha-n} x(s) d s, \\
T_{4} y= & \int_{0}^{1}(1-s)^{\beta-n} y(s) d s-\frac{\delta_{2}}{\beta-n+1} \int_{0}^{\eta_{2}}\left(\eta_{2}-s\right)^{\beta-n+1} y(s) d s \\
& -\gamma_{2} \delta_{2} \int_{0}^{\xi_{2}}\left(\xi_{2}-s\right)^{\beta-n} y(s) d s .
\end{aligned}
$$

For $\forall t \in[0,1]$, the linear continuous projector operators $P: \bar{X} \rightarrow \bar{X}$ and $Q: \bar{Y} \rightarrow \bar{Y}$ can be defined as

$$
\begin{aligned}
& P(u, v)=\left(P_{1} u, P_{2} v\right)=\left(\frac{u^{(n-1)}(0)}{(n-1) !}\left(t^{n-1}+(n-1) \gamma_{1} t^{n-2}\right), \frac{v^{(n-1)}(0)}{(n-1) !}\left(t^{n-1}+(n-1) \gamma_{2} t^{n-2}\right)\right), \\
& Q(x, y)=\left(Q_{1} x, Q_{2} y\right)=\left(\Lambda_{1} T_{3} x, \Lambda_{2} T_{4} y\right),
\end{aligned}
$$


where

$$
\Lambda_{1}=\frac{\alpha-n+1}{1-\frac{\delta_{1}}{\alpha-n+2} \eta_{1}^{\alpha-n+2}-\gamma_{1} \delta_{1} \xi_{1}^{\alpha-n+1}}, \quad \Lambda_{2}=\frac{\beta-n+1}{1-\frac{\delta_{2}}{\beta-n+2} \eta_{2}^{\beta-n+2}-\gamma_{2} \delta_{2} \xi_{2}^{\beta-n+1}} .
$$

Define the operator $K_{P}: \operatorname{Im} L \rightarrow \operatorname{dom} L \cap \operatorname{Ker} P$ as

$$
\begin{aligned}
K_{P}(x, y)= & \left(\frac{1}{\Gamma(\alpha)} \int_{0}^{t}(t-s)^{\alpha-1} x(s) d s+\frac{\gamma_{1} t^{n-2}}{(n-2) ! \Gamma(\alpha-n+1)} \int_{0}^{\xi_{1}}\left(\xi_{1}-s\right)^{\alpha-n} x(s) d s,\right. \\
& \left.\frac{1}{\Gamma(\beta)} \int_{0}^{t}(t-s)^{\beta-1} y(s) d s+\frac{\gamma_{2} t^{n-2}}{(n-2) ! \Gamma(\beta-n+1)} \int_{0}^{\xi_{2}}\left(\xi_{2}-s\right)^{\beta-n} y(s) d s\right) .
\end{aligned}
$$

Next, we give the proof of Theorem 3.2 (similar to Theorem 3.1).

Proof Firstly, it will be proved that the set

$$
\Omega_{1}=\{(u, v) \in \operatorname{dom} L \backslash \operatorname{Ker} L \mid L(u, v)=\lambda N(u, v), \lambda \in(0,1)\}
$$

is bounded. If $(u, v) \in \Omega_{1}$, similar to Step 1 in the proof of Theorem 3.1, we get

$$
\left|u^{(n-1)}(0)\right| \leq a_{1}\left(\sum_{i=0}^{n-1}\left\|a_{i}\right\|_{\infty}\left\|v^{(i)}\right\|_{\infty}+\left\|b_{1}\right\|_{\infty} \sum_{i=0}^{n-1}\left\|v^{(i)}\right\|_{\infty}^{\theta_{1}}+\left\|r_{1}\right\|_{\infty}\right)+M
$$

where $a_{1}=\frac{1}{\Gamma(\alpha-n+2)}$, and

$$
\left|\nu^{(n-1)}(0)\right| \leq a_{2}\left(\sum_{i=0}^{n-1}\left\|d_{i}\right\|_{\infty}\left\|u^{(i)}\right\|_{\infty}+\left\|b_{2}\right\|_{\infty} \sum_{i=0}^{n-1}\left\|u^{(i)}\right\|_{\infty}^{\theta_{2}}+\left\|r_{2}\right\|_{\infty}\right)+M
$$

where $a_{2}=\frac{1}{\Gamma(\beta-n+2)}$.

So

$$
\|P(u, v)\|_{\bar{X}} \leq \max \left\{\left(1+(n-1) \gamma_{1}\right)\left|u^{(n-1)}(0)\right|,\left(1+(n-1) \gamma_{2}\right)\left|v^{(n-1)}(0)\right|\right\} .
$$

On the other hand, for $(x, y) \in \operatorname{Im} L$, by the definition of $\|\cdot\|_{\bar{X}}$ and $K_{P}$, it is easy to see that

$$
\left\|K_{P}(x, y)\right\|_{\bar{X}} \leq \max \left\{\left(1+\gamma_{1}\right) a_{1}\|x\|_{\infty},\left(1+\gamma_{2}\right) a_{2}\|y\|_{\infty}\right\} .
$$

Hence,

$$
\begin{aligned}
\|(I-P)(u, v)\|_{\bar{X}} & =\left\|K_{P} L(I-P)(u, v)\right\|_{\bar{X}}=\left\|K_{P} L(u, v)\right\|_{\bar{X}}=\left\|K_{P}\left(L_{1} u, L_{2} v\right)\right\|_{\bar{X}} \\
& \leq \max \left\{\left(1+\gamma_{1}\right) a_{1}\left\|N_{1} v\right\|_{\infty},\left(1+\gamma_{2}\right) a_{2}\left\|N_{2} u\right\|_{\infty}\right\} .
\end{aligned}
$$


Thus,

$$
\begin{aligned}
\|(u, v)\|_{\bar{X}}= & \|P(u, v)+(I-P)(u, v)\|_{\bar{X}} \\
\leq & \|P(u, v)\|_{\bar{X}}+\|(I-P)(u, v)\|_{\bar{X}} \\
\leq & \max \left\{\left(1+(n-1) \gamma_{1}\right)\left|u^{(n-1)}(0)\right|+\left(1+\gamma_{1}\right) a_{1}\left\|N_{1} v\right\|_{\infty},\right. \\
& \left(1+(n-1) \gamma_{1}\right)\left|u^{(n-1)}(0)\right|+\left(1+\gamma_{2}\right) a_{2}\left\|N_{2} u\right\|_{\infty}, \\
& \left(1+(n-1) \gamma_{2}\right)\left|v^{(n-1)}(0)\right|+\left(1+\gamma_{1}\right) a_{1}\left\|N_{1} v\right\|_{\infty}, \\
& \left.\left(1+(n-1) \gamma_{2}\right)\left|v^{(n-1)}(0)\right|+\left(1+\gamma_{2}\right) a_{2}\left\|N_{2} u\right\|_{\infty}\right\} .
\end{aligned}
$$

Next we will prove this conclusion in four cases.

Case $1^{\prime}\|(u, v)\|_{\bar{X}} \leq\left(1+(n-1) \gamma_{1}\right)\left|u^{(n-1)}(0)\right|+\left(1+\gamma_{1}\right) a_{1}\left\|N_{1} v\right\|_{\infty}$. By (3.7) and $\left(\mathrm{H}_{1}\right)^{\prime}$, we get

$$
\begin{aligned}
\|(u, v)\|_{\bar{X}} \leq & \left(1+(n-1) \gamma_{1}\right) a_{1}\left(\sum_{i=0}^{n-1}\left\|a_{i}\right\|_{\infty}\left\|v^{(i)}\right\|_{\infty}+\left\|b_{1}\right\|_{\infty} \sum_{i=0}^{n-1}\left\|v^{(i)}\right\|_{\infty}^{\theta_{1}}+\|r\|_{\infty}\right) \\
& +\left(1+(n-1) \gamma_{1}\right) M+\left(1+\gamma_{1}\right) a_{1}\left\|f\left(t, v(t), v^{\prime}(t), \ldots, v^{(n-1)}(t)\right)\right\|_{\infty} \\
\leq & \left(2+n \gamma_{1}\right) a_{1}\left(\sum_{i=0}^{n-1}\left\|a_{i}\right\|_{\infty}\left\|v^{(i)}\right\|_{\infty}+\left\|b_{1}\right\|_{\infty} \sum_{i=0}^{n-1}\left\|v^{(i)}\right\|_{\infty}^{\theta_{1}}+\left\|r_{1}\right\|_{\infty}\right) \\
& +\left(1+(n-1) \gamma_{1}\right) M \\
\leq & \left(2+n \gamma_{1}\right) a_{1}\left(\sum_{i=0}^{n-1}\left\|a_{i}\right\|_{\infty}\left\|v^{(n-1)}\right\|_{\infty}+n\left\|b_{1}\right\|_{\infty}\left\|v^{(n-1)}\right\|_{\infty}^{\theta_{1}}+\left\|r_{1}\right\|_{\infty}\right) \\
& +\left(1+(n-1) \gamma_{1}\right) M \\
= & \left(2+n \gamma_{1}\right) a_{1}\left(\sum_{i=0}^{n-1}\left\|a_{i}\right\|_{\infty}\|v\|_{X}+n\left\|b_{1}\right\|_{\infty}\|v\|_{\infty}^{\theta_{1}}+\left\|r_{1}\right\|_{\infty}\right)+\left(1+(n-1) \gamma_{1}\right) M
\end{aligned}
$$

According to $\left(\mathrm{H}_{4}\right)^{\prime}$ and the definition of $\|(u, v)\|_{\bar{X}}$, we see that $\|v\|_{X}$ is bounded, therefore $\Omega_{1}$ is bounded.

Case $2^{\prime}\|(u, v)\|_{\bar{X}} \leq\left|v^{(n-1)}(0)\right|+a_{2}\left\|N_{2} u\right\|_{\infty}$. The proof is similar to Case $1^{\prime}$. Here, we omit it.

Case $3^{\prime}\|(u, v)\|_{\bar{X}} \leq\left|u^{(n-1)}(0)\right|+a_{2}\left\|N_{2} u\right\|_{\infty}$. By (3.7) and $\left(\mathrm{H}_{1}\right)^{\prime}$, we get

$$
\begin{aligned}
\|(u, v)\|_{\bar{X}} \leq & \left|u^{(n-1)}(0)\right|+a_{2}\left\|N_{2} u\right\|_{\infty} \\
\leq & a_{1}\left(\sum_{i=0}^{n-1}\left\|a_{i}\right\|_{\infty}\left\|v^{(i)}\right\|_{\infty}+\left\|b_{1}\right\|_{\infty} \sum_{i=0}^{n-1}\left\|v^{(i)}\right\|_{\infty}^{\theta_{1}}+\left\|r_{1}\right\|_{\infty}\right)+M \\
& +a_{2}\left\|g\left(t, u(t), u^{\prime}(t), \ldots, u^{(n-1)}(t)\right)\right\|_{\infty} \\
\leq & a_{1}\left(\sum_{i=0}^{n-1}\left\|a_{i}\right\|_{\infty}\left\|v^{(i)}\right\|_{\infty}+\left\|b_{1}\right\|_{\infty} \sum_{i=0}^{n-1}\left\|v^{(i)}\right\|_{\infty}^{\theta_{1}}+\left\|r_{1}\right\|_{\infty}\right)+M \\
& +a_{2}\left(\sum_{i=0}^{n-1}\left\|d_{i}\right\|_{\infty}\left\|u^{(i)}\right\|_{\infty}+\left\|b_{2}\right\|_{\infty} \sum_{i=0}^{n-1}\left\|u^{(i)}\right\|_{\infty}^{\theta_{2}}+\left\|r_{2}\right\|_{\infty}\right)
\end{aligned}
$$




$$
\begin{aligned}
\leq & a_{1}\left(\sum_{i=0}^{n-1}\left\|a_{i}\right\|_{\infty}\left\|v^{(n-1)}\right\|_{\infty}+n\left\|b_{1}\right\|_{\infty}\left\|v^{(n-1)}\right\|_{\infty}^{\theta_{1}}+\left\|r_{1}\right\|_{\infty}\right)+M \\
& +a_{2}\left(\sum_{i=0}^{n-1}\left\|d_{i}\right\|_{\infty}\left\|u^{(n-1)}\right\|_{\infty}+n\left\|b_{2}\right\|_{\infty}\left\|u^{(n-1)}\right\|_{\infty}^{\theta_{2}}+\left\|r_{2}\right\|_{\infty}\right) \\
= & a_{1}\left(\sum_{i=0}^{n-1}\left\|a_{i}\right\|_{\infty}\|v\|_{X}+n\left\|b_{1}\right\|_{\infty}\|v\|_{X}^{\theta_{1}}+\left\|r_{1}\right\|_{\infty}\right) \\
& +a_{2}\left(\sum_{i=0}^{n-1}\left\|d_{i}\right\|_{\infty}\|u\|_{X}+n \mid b_{2}\left\|_{\infty}\right\| u\left\|_{X}^{\theta_{2}}+\right\| r_{2} \|_{\infty}\right)+M .
\end{aligned}
$$

By $\left(\mathrm{H}_{4}\right)^{\prime}$, we get $\|(u, v)\|_{\bar{X}}$ is bounded, therefore $\Omega_{1}$ is bounded.

Case $4^{\prime}\|(u, v)\|_{\bar{X}} \leq\left|v^{(n-1)}(0)\right|+a_{1}\left\|N_{1} v\right\|_{\infty}$. The proof is similar to Case $3^{\prime}$. Here, we omit it.

In summary, we proved that $\Omega_{1}$ is bounded. The remainder of the proof is just similar to the proof of Theorem 3.1 and is omitted.

\section{Example}

Example 4.1 Consider the following BVP:

$$
\begin{cases}D_{0+}^{3.5} u(t)=f\left(t, v, v^{\prime}, v^{\prime \prime}, v^{\prime \prime \prime}\right), & 0<t<1, \\ D_{0+}^{3.6} v(t)=g\left(t, u, u^{\prime}, u^{\prime \prime}, u^{\prime \prime \prime}\right), & 0<t<1, \\ u(0)=u^{\prime}(0)=u^{\prime \prime}(0)=0, & u^{\prime \prime \prime}(1)=4 u^{\prime \prime}\left(\frac{1}{4}\right) \\ v(0)=v^{\prime}(0)=v^{\prime \prime}(0)=0, & v^{\prime \prime \prime}(1)=5 v^{\prime \prime}\left(\frac{1}{5}\right)\end{cases}
$$

where $\alpha=3.5, \beta=3.6, f\left(t, x_{0}, x_{1}, x_{2}, x_{3}\right)=\frac{x_{0} \cos t+x_{1} \sin t+x_{2}}{8}+\frac{x_{3}}{32}+\sin \left(x_{0} x_{2}+t x_{1}\right), g\left(t, y_{0}, y_{1}\right.$, $\left.y_{2}, y_{3}\right)=\frac{t}{7}+\frac{y_{0} \sin 2 t}{16}+\frac{y_{1} \cos 3 t}{9}+\frac{y_{2}+y_{3}}{8}$, it is easily figured out that $\gamma_{1}=\gamma_{2}=0, \delta_{1}=4, \delta_{2}=5$, $\eta_{1}=\frac{1}{4}, \eta_{2}=\frac{1}{5}$, satisfy the condition of Case (i), $\left\|a_{0}\right\|_{\infty}=\left\|a_{1}\right\|_{\infty}=\left\|a_{2}\right\|_{\infty}=\frac{1}{8},\left\|a_{3}\right\|_{\infty}=\frac{1}{32}$, $\left\|d_{0}\right\|_{\infty}=\frac{1}{16},\left\|d_{1}\right\|_{\infty}=\frac{1}{9},\left\|d_{2}\right\|_{\infty}=\left\|d_{3}\right\|_{\infty}=\frac{1}{8},\left\|b_{1}\right\|_{\infty}=\left\|b_{2}\right\|_{\infty}=0,\left\|r_{1}\right\|_{\infty}=1,\left\|r_{2}\right\|_{\infty}=\frac{1}{7}$, $\theta_{1}=\theta_{2}=0, M=64, M^{*}=32,0<t<1$, it is easy to verify that the conditions satisfy all assumptions of Theorem 3.1. Hence, BVP (4.1) has at least one set of solutions.

Example 4.2 Consider the BVP:

$$
\left\{\begin{array}{l}
D_{0+}^{3.3} u(t)=f\left(t, v, v^{\prime}, v^{\prime \prime}, v^{\prime \prime \prime}\right), \quad 0<t<1, \\
D_{0+}^{3.5} v(t)=g\left(t, u, u^{\prime}, u^{\prime \prime}, u^{\prime \prime \prime}\right), \quad 0<t<1, \\
u(0)=u^{\prime}(0)=0, \quad u^{\prime \prime}(0)=3 u^{\prime \prime \prime}\left(\frac{1}{3}\right), \quad u^{\prime \prime \prime}(1)=\frac{2}{7} u^{\prime \prime}\left(\frac{1}{2}\right), \\
v(0)=v^{\prime}(0)=0, \quad v^{\prime \prime}(0)=2 v^{\prime \prime \prime}\left(\frac{1}{2}\right), \quad v^{\prime \prime \prime}(1)=\frac{3}{7} v^{\prime \prime}\left(\frac{1}{3}\right),
\end{array}\right.
$$

where $\alpha=3.3, \beta=3.5, f\left(t, x_{0}, x_{1}, x_{2}, x_{3}\right)=\frac{x_{0} \sin t+x_{1} \cos t+x_{2}}{56}+\frac{x_{3}}{64}+\cos \left(x_{1}+t x_{2}\right), g\left(t, y_{0}, y_{1}\right.$, $\left.y_{2}, y_{3}\right)=\frac{t}{6}+\frac{y_{0} \sin (3 t+1)}{30}+\frac{y_{1} \cos (2 t+4)}{45}+\frac{y_{2}+y_{3}}{50}$, it is easily figured out that $\gamma_{1}=3, \gamma_{2}=2, \delta_{1}=\frac{2}{7}$, $\delta_{2}=\frac{3}{7}, \eta_{1}=\frac{1}{2}, \eta_{2}=\frac{1}{3}$ satisfy the condition of Case (ii), $\left\|a_{0}\right\|_{\infty}=\left\|a_{1}\right\|_{\infty}=\left\|a_{2}\right\|_{\infty}=\frac{1}{56}$, $\left\|a_{3}\right\|_{\infty}=\frac{1}{64},\left\|d_{0}\right\|_{\infty}=\frac{1}{30},\left\|d_{1}\right\|_{\infty}=\frac{1}{45},\left\|d_{2}\right\|_{\infty}=\left\|d_{3}\right\|_{\infty}=\frac{1}{50},\left\|b_{1}\right\|_{\infty}=\left\|b_{2}\right\|_{\infty}=0,\left\|r_{1}\right\|_{\infty}=1$, $\left\|r_{2}\right\|_{\infty}=\frac{1}{6}, \theta_{1}=\theta_{2}=0, M=120, M^{*}=90,0<t<1$, it is easy to verify that the conditions satisfy all assumptions of Theorem 3.2. Hence, BVP (4.2) has at least one set of solutions. 


\section{Acknowledgements}

This work is supported by the National Natural Science Foundation of China (11271364).

\section{Competing interests}

The authors declare that they have no competing interests.

\section{Authors' contributions}

The authors contributed equally in this article. They all read and approved the final manuscript.

\section{Author details}

'Department of Mathematics, China University of Mining and Technology, Xuzhou, 221116, P.R. China. ${ }^{2}$ Department of Basic, Xinjiang Institute of Engineering, Urumqi, 830000, P.R. China.

\section{Publisher's Note}

Springer Nature remains neutral with regard to jurisdictional claims in published maps and institutional affiliations.

Received: 12 May 2017 Accepted: 30 August 2017 Published online: 29 September 2017

\section{References}

1. Leszczynski, JS, Blaszczyk, T: Modeling the transition between stable and unstable operation while emptying a silo. Granul. Matter 13, 429-438 (2011)

2. Magin, RL: Fractional calculus models of complex dynamics in biological tissues. Comput. Math. Appl. 59, 1586-1593 (2010)

3. Kai, D, Ford, NJ, Freed, AD: A predictor-corrector approach for the numerical solution of fractional differential equations. Nonlinear Dyn. 29, 3-22 (2002)

4. Szymanek, E: The application of fractional order differential calculus for the description of temperature profiles in a granular layer. Advances in the Theory and Applications of Non-integer Order Systems 257, $243-248$ (2013)

5. Ahmad, B, Agarwal, RP: Some new versions of fractional boundary value problems with slit-strips conditions. Bound. Value Probl. 2014, 175 (2014)

6. Li, M, Wang, J: Finite time stability of fractional delay differential equations. Appl. Math. Lett. 64, 170-176 (2017)

7. Wang, J, Fečkan, M, Zhou, Y: Center stable manifold for planar fractional damped equations. Appl. Math. Comput. 296, 257-269 (2017)

8. Wei, Z, Li, Q, Che, J: Periodic boundary value problems for fractional differential equations involving a Riemann-Liouville fractional derivative. Nonlinear Anal. 73, 3232-3238 (2010)

9. Herzallah, M, Baleanu, D: Existence of a periodic mild solution for a nonlinear fractional differential equation. Comput. Math. Appl. 64, 3059-3064 (2012)

10. $\mathrm{Hu}, \mathrm{Z}, \mathrm{Liu}, \mathrm{W}, \mathrm{Rui}, \mathrm{W}:$ Periodic boundary value problem for fractional differential equation. Int. J. Math. 23, 1-11 (2012)

11. Agarwal, RP, Ahmad, B: Existence theory for anti-periodic boundary value problems of fractional differential equations and inclusions. Comput. Math. Appl. 62, 1200-1214 (2011)

12. Sun, J, Liu, Y, Liu, G: Existence of solutions for fractional differential systems with antiperiodic boundary conditions. Comput. Math. Appl. 64, 1557-1566 (2012)

13. Wang, M: A fractional boundary value problem with Dirichlet boundary conditions. Commun. Appl. Anal. 19, 497-504 (2014)

14. Nyamoradi, N: Infinitely many solutions for a class of fractional boundary value problems with Dirichlet boundary conditions. Mediterr. J. Math. 11, 75-87 (2014)

15. Bai, Z, Zhang, Y: Solvability of fractional three-point boundary value problems with nonlinear growth. Appl. Math Comput. 218, 1719-1725 (2011)

16. Liu, Y: Solvability of multi-point boundary value problems for multiple term Riemann-Liouville fractional differential equations. Comput. Math. Appl. 64, 413-431 (2012)

17. Bai, Z: Solvability for a class of fractional m-point boundary value problem at resonance. Comput. Math. Appl. 62 , 1292-1302 (2011)

18. Wang, J, Fečkan, M, Zhou, Y: A survey on impulsive fractional differential equations. Fract. Calc. Appl. Anal. 19, 806-831 (2016)

19. Yang, X: Studies on Sturm-Liouville boundary value problems for multi-term fractional differential equations. Comput. Math. Appl. 64, 413-431 (2012)

20. Zhang, L, Ge, W: Solvability of a kind of Sturm-Liouville boundary value problems with impulses via variational methods. Acta Appl. Math. 110, 1237-1248 (2010)

21. Al-Mdallal, QM: On the numerical solution of fractional Sturm-Liouville problems. Int. J. Comput. Math. 87, 2837-2845 (2010)

22. Ertürk, VS: Computing eigenelements of Sturm-Liouville problems of fractional order via fractional differential transform method. Math. Comput. Appl. 16, 712-720 (2011)

23. Zhao, X, Chai, C, Ge, W: Positive solutions for fractional four-point boundary value problems. Commun. Nonlinear Sci. Numer. Simul. 16, 3665-3672 (2011)

24. Liu, Y: Application of Avery-Peterson fixed point theorem to nonlinear boundary value problem of fractional differential equation with the Caputo's derivative. Commun. Nonlinear Sci. Numer. Simul. 17, 4576-4584 (2012)

25. Zhao, Y, Yang, L, Chen, P: Positive solutions to boundary value problems of a high-order fractional differential equation in a Banach space. Adv. Differ. Equ. 2013, 344 (2013)

26. Deng, WH, Li, CP: Chaos synchronization of the fractional Lü system. Physica A 353, 61-72 (2005)

27. Chen, $Y, A n, H L$ : Numerical solutions of coupled Burgers equations with time- and space-fractional derivatives. Appl. Math. Comput. 200, 87-95 (2008) 
28. Gafiychuk, V, Datsko, B, Meleshko, V: Mathematical modeling of time fractional reaction-diffusion systems. J. Comput. Appl. Math. 220, 215-225 (2008)

29. Khodabakhshi, N, Vaezpour, SM: Existence results for a coupled system of nonlinear fractional differential equations with boundary value problems on an unbounded domain. Electron. J. Qual. Theory Differ. Equ. 2013, 73 (2013)

30. Zou, Y, Liu, L, Cui, Y: The existence of solutions for four-point coupled boundary value problems of fractional differential equations at resonance. Abstr. Appl. Anal. 2014, 314083 (2014)

31. Zhai, C, Hao, M: Multi-point boundary value problems for a coupled system of nonlinear fractional differential equations. Adv. Differ. Equ. 2015, 147 (2015)

32. Hu, Z, Liu, W: Solvability of a coupled system of fractional differential equations with periodic boundary conditions at resonance. Ukr. Math. J. 65, 1619-1633 (2014)

33. Zhang, Y, Bai, Z, Feng, T: Existence results for a coupled system of nonlinear fractional three-point boundary value problems at resonance. Comput. Math. Appl. 61, 1032-1047 (2011)

34. Hu, L, Zhang, S: Existence and uniqueness of solutions for a higher-order coupled fractional differential equations at resonance. Adv. Differ. Equ. 2015, 202 (2015)

35. Bashir, A, Ahmed, A: Existence and uniqueness of solutions for coupled systems of higher-order nonlinear fractional differential equations. Fixed Point Theory Appl. 2010, Article ID 364560 (2010)

36. Feng, $\mathrm{M}, \mathrm{Zhang}, \mathrm{X}, \mathrm{Ge}, \mathrm{W}$ : New existence results for higher-order nonlinear fractional differential equation with integral boundary conditions. Bound. Value Probl. 2011, Article ID 720702 (2011)

37. Wang, L, Zhang, X: Existence of positive solutions for a class of higher-order nonlinear fractional differential equations with integral boundary conditions and a parameter. J. Appl. Math. Comput. 44, 293-316 (2014)

38. Zhao, K, Gong, P: Existence of positive solutions for a class of higher-order Caputo fractional differential equation. Qual. Theory Dyn. Syst. 14, 157-171 (2015)

39. Mawhin, J: Topological Degree and Boundary Value Problems for Nonlinear Differential Equations. Topological Methods for Ordinary Differential Equations. Springer, Berlin (1993)

40. Kilbas, AA, Srivastava, HM, Trujillo, JJ: Theory and Applications of Fractional Differential Equations. North-Holland Mathematics Studies Elsevier, Amsterdam (2006)

41. Podlubny, I: Fractional Differential Equations. Mathematics in Science and Engineering. Academic Press, New York (1999)

\section{Submit your manuscript to a SpringerOpen ${ }^{\circ}$ journal and benefit from:}

- Convenient online submission

- Rigorous peer review

- Open access: articles freely available online

- High visibility within the field

Retaining the copyright to your article

Submit your next manuscript at $\boldsymbol{~ s p r i n g e r o p e n . c o m ~}$ 